\title{
Delivery of MSCs with a Hybrid $\beta$-Sheet Peptide Hydrogel Consisting IGF-IC Domain and D-Form Peptide for Acute Kidney Injury Therapy
}

This article was published in the following Dove Press journal: International Journal of Nanomedicine

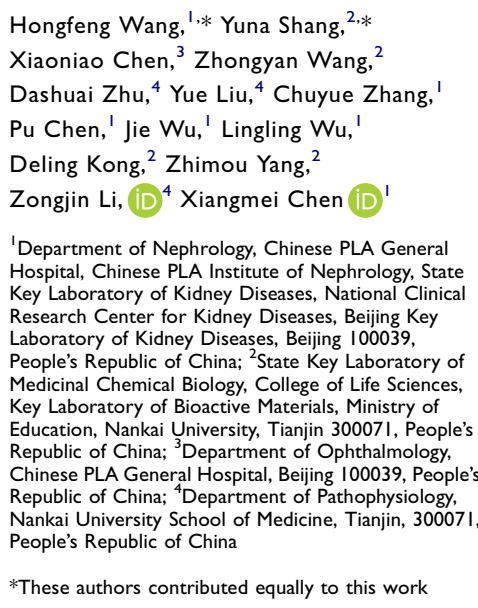

Hongfeng Wang, ${ }^{1, *}$ Yuna Shang, ${ }^{2, *}$ Xiaoniao Chen, ${ }^{3}$ Zhongyan Wang, ${ }^{2}$ Dashuai Zhu, ${ }^{4}$ Yue Liu, ${ }^{4}$ Chuyue Zhang, Pu Chen,' Jie Wu,' Lingling Wu,' Deling Kong, ${ }^{2}$ Zhimou Yang, ${ }^{2}$ Zongjin Li, (iD ${ }^{4}$ Xiangmei Chen (iD)

'Department of Nephrology, Chinese PLA General Hospital, Chinese PLA Institute of Nephrology, State Key Laboratory of Kidney Diseases, National Clinical Research Center for Kidney Diseases, Beijing Key Laboratory of Kidney Diseases, Beijing 100039 , People's Republic of China; ${ }^{2}$ State Key Laboratory of Medicinal Chemical Biology, College of Life Sciences, Medicinal Chemical Biology, College of Life Sciences, Key Laboratory of Bioactive Materials, Ministry of Education, Nankai University, Tianjin 30007I, People's Republic of China; ' $D$ Department of Ophthalmology, Chinese PLA General Hospital, Beijing 100039, People' Republic of China; ${ }^{4}$ Department of Pathophysiology, Nankai University School of Medicine, Tianjin, 30007I, People's Republic of China

*These authors contributed equally to this work

\section{Video abstract}

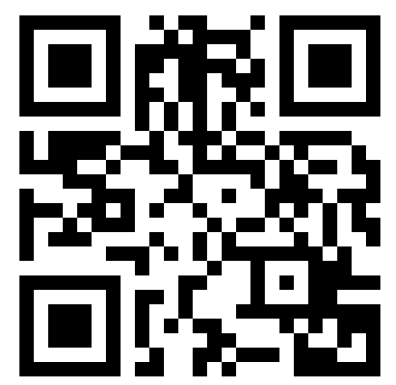

Point your SmartPhone at the code above. If you have a $Q R$ code reader the video abstract will appear. Or use: https://youtu.be/Zlks I7GICP4

Correspondence: Xiangmei Chen

Chinese PLA General Hospital, Beijing 100039. People's Republic of China

Email xmchen30I@I26.com

Zhimou Yang

Nankai University, Tianjin 30007I, People's

Republic of China

Email yangzm@nankai.edu.cn
Purpose: By providing a stem cell microenvironment with particular bioactive constituents in vivo, synthetic biomaterials have been progressively successful in stem cell-based tissue regeneration by enhancing the engraftment and survival of transplanted cells. Designs with bioactive motifs to influence cell behavior and with D-form amino acids to modulate scaffold stability may be critical for the development and optimization of self-assembling biomimetic hydrogel scaffolds for stem cell therapy.

Materials and Methods: In this study, we linked naphthalene (Nap) covalently to a short D-form peptide (Nap- ${ }^{D} F^{D} F G$ ) and the $\mathrm{C}$ domain of insulin-like growth factor-1 (IGF-1C) as a functional hydrogel-based scaffolds, and we hypothesized that this hydrogel could enhance the therapeutic efficiency of human placenta-derived mesenchymal stem cells (hP-MSCs) in a murine acute kidney injury (AKI) model.

Results: The self-assembling peptide was constrained into a classical $\beta$-sheet structure and showed hydrogel properties. Our results revealed that this hydrogel exhibited increased affinity for IGF-1 receptor. Furthermore, cotransplantation of the $\beta$-IGF-1C hydrogel and $\mathrm{hP}-\mathrm{MSCs}$ contributed to endogenous regeneration post-injury and boosted angiogenesis in a murine AKI model, leading to recovery of renal function.

Conclusion: This hydrogel could provide a favorable niche for hP-MSCs and thereby rescue renal function in an AKI model by promoting cell survival and angiogenesis. In conclusion, by covalently linking the desired functional groups to D-form peptides to create functional hydrogels, self-assembling $\beta$-sheet peptide hydrogels may serve as a promising platform for tissue-engineering and stem cell therapy.

Keywords: self-assembly, $\beta$-sheet, hydrogel, D-form peptide, C domain of insulin-like growth factor, IGF-1C, mesenchymal stem cells, MSCs, acute kidney injure, AKI

\section{Introduction}

The utilization of biomaterials to mimic the extracellular matrix (ECM) provides cells with structural support and biochemical cues in stem cell-based therapies. ${ }^{1-3}$ Self-assembling peptides, which self-organize from naturally building blocks into supramolecular nanofibrous structures, can be used to recreate native stem cell microenvironments in vivo. ${ }^{4,5}$ The initial and rational design for self-assembling peptides was inspired by $\beta$-sheet and $\alpha$-helical structures found in naturally occurring proteins. ${ }^{2,4,6,7}$ Insights into peptide folding and intramolecular interactions that drive self-assembly have led to the emergence of customized de novo motifs. Hydrogels with $\beta$-sheet structures are of particular interest because peptides can 
adopt a $\beta$-sheet conformation that undergoes a dynamic reassembly process without affecting the bulk structural property. ${ }^{8,9}$ Moreover, by covalently linking a desired functional group to the peptide, a hydrogel can incorporate additional functionalities and properties for tissueengineering ${ }^{10}$.

Numerous designs of self-assembling hydrogels with $\beta$-sheet structures have been identified to promote cell growth, proliferation and differentiation. ${ }^{4,7}$ To develop specific cell-scaffolds for tissue regeneration, biologically active motifs for cell attachment can be chemically conjugated to create functional hydrogels through selfassembly. ${ }^{3,10-13}$ As bulky proteins have disadvantages in terms of immunogenicity and sudden loss of bioactivity, accumulating evidence supports the use of bioactivitymimicking peptides such as RGD, QK and QHREDGS, as surrogates for fibronectin, vessel endothelial growth factor (VEGF), and angiopoietin-1, respectively, to increase the bioactivity of engineered matrices. ${ }^{14-16}$ The 12-peptide sequence GYGSSSRRAPQT, known as the C domain of insulin-like growth factor-1 (IGF-1C), has been identified as the active region of the IGF-1 protein, which can increase stem cell survival and substantially improve renal function in acute kidney injury (AKI) ${ }^{17,18}$.

The kidney has the capacity to regenerate and repair itself provided that the damage is limited and the structure remains intact. ${ }^{19-22}$ However, this potential may be hindered due to excessive damage and maladapted repair, leading to a progression of chronic kidney disease (CKD) along with a need for renal replacement therapy. ${ }^{23}$ With the ability to proliferate and differentiate into a wide variety of lineages, stem cells, such as mesenchymal stem cells (MSCs), have been used for kidney regeneration. ${ }^{17}$ Unfortunately, poor cell survival and engraftment after transplantation limits the successful use of cellular therapy for AKI or CKD. ${ }^{17,23}$ Recent advances in the field of biomaterials have provided synthetic 3D ECM by mimicking the components of tissue microenvironments to prevent implanted stem cell apoptosis by triggering specific signal pathways. ${ }^{24,25}$

Amino acids are all chiral, with the exception of glycine. Self-assembling peptides containing D-amino acids are more stable because natural proteases can readily degrade L-form peptide bonds, but cannot degrade D-form peptide bonds. ${ }^{26-29}$ A recent study revealed that self-assembling peptide hydrogels with $\beta$-sheet structures, made of D-amino acids, exhibit high resistance against enzymatic hydrolysis. ${ }^{30}$ The conjugation of bioactivity-mimicking peptides to the backbone of self- assembling $\beta$-sheets derived from D-amino acids may result in improved biostability and bioactivity for potential therapeutic applications. ${ }^{28,30,31}$ This solution may provide a more effective way to generate hydrogel scaffolds for stem cells in regenerative medicine.

In this study, we first introduced $\mathrm{D}$-amino acids into the self-assembly motif Nap-FFG (Nap- ${ }^{\mathrm{D}} \mathrm{F}^{\mathrm{D}} \mathrm{FG}$ ) to improve enzymatic stability ${ }^{28,29,32}$ and we then covalently attached the Nap- ${ }^{\mathrm{D}} \mathrm{F}^{\mathrm{D}} \mathrm{FG}$ peptide to the IGF-1C domain to yield a supramolecular self-assembly peptide. This de novo peptide adopts a typical $\beta$-sheet conformation via heatingcooling methods, and demonstrates increased affinity for IGF-1 receptors, mediating superior bioactivity. We further investigated whether this hydrogel could provide a favorable niche for human placenta-derived mesenchymal stem cells (hP-MSCs) and thereby enhance the therapeutic efficiency of hP-MSCs in an AKI model.

\section{Materials and Methods Materials}

All chemicals and reagents were purchased from SigmaAldrich (Missouri, St. Louis) unless otherwise indicated. Dulbecco's modified Eagle's medium (DMEM)/F12, minimum essential medium (MEM) and nonessential amino acid (NEAA) solution were purchased from Gibco (Grand Island, NY). Endothelial Cell Growth Medium-2 (EGM-2) was purchased from Lonza (Basel, Switzerland). Fetal bovine serum (FBS) was purchased from HyClone (Australia). A penicillin-streptomycin solution was purchased from Gibco (Rockville, MD). Matrigel was purchased from Corning (NY, USA). D-Luciferin was purchased from Biosynth International (Biosynth International, Naperville, IL). The radio immunoprecipitation assay (RIPA) buffer was purchased from Solarbio (Shanghai, China). The phenylmethanesulfonyl fluoride (PMSF) was purchased from Roche Molecular BioChemicals (Indianapolis, IN). The BCA protein assay Kit was purchased from Promega (Madison, WI). The polyvinylidene fluoride (PVDF) membranes were purchased from Millipore (Watford, UK). The primary antibodies anti-Ki-67 (1:200, DAKO, M7268), anti-kidney injury molecule-1 (KIM-1) (1:200, SANTA, 166,785), anti-von Willebrand factor (vWF) (1:200, Abcam, ab6586), anti-collagen IV (1:200, Abcam, ab154193), Lotus tetragonolobus lectin (LTL) (1:200, Vector, fl-1321), anti-IGF-1 receptor $\beta$ (1:1000, CST, D23H3), anti-phospho-IGF-1 receptor $\beta$ (1:1000, CST, 3021) and anti-tubulin (1:1000, Abcam, EPR13796) were used in the corresponding analyses. 


\section{Peptide Synthesis and Purification}

All peptides were prepared by standard solid phase peptide synthesis (SPPS) by using 2-chlorotrityl chloride resin and the corresponding N-Fmoc (9-fluorenylmethyl carbamates) protected amino acids with the side chains properly protected. ${ }^{11,12}$ First, the C-terminus of the first amino acid was conjugated on the resin. Anhydrous N, N'dimethyl formamide (DMF) containing 20\% piperidine was used to remove the Fmoc protection group. To couple the next amino acid to the free amino group, O-benzotriazol-1-yl-N, N, N', N'-tetramethyluronium hexafluorophosphate (HBTU) was used as the coupling reagent. The peptide chain was extended according to the standard SPPS protocol. Finally, 95\% trifluoroacetic acid (TFA) containing $2.5 \% \mathrm{H}_{2} \mathrm{O}$ and $2.5 \%$ triisopropyl silane (TIS) was used to cleave peptide derivatives from the resin and the mixture was filtered. Anhydrous diethyl ether was added to the filtrate concentrated by rotary evaporation. The precipitate was left to rest for 4 hours before being dried with vacuum pump and then purified by high-performance liquid chromatography (HPLC) to obtain pure compounds.

\section{Preparation of Hydrogels}

Preparation of the hydrogel followed the heating-cooling protocol described previously. ${ }^{11}$ In brief, $2.5 \mathrm{mg}$ of lyophilized self-assembling IGF-1C was dissolved in $496 \mu \mathrm{L}$ of phosphate buffered solution (PBS), and $4 \mu \mathrm{L}$ of $1 \mathrm{M}$ sodium carbonate was added to adjust the $\mathrm{pH}$ to 7.4. The mixture was heated with an alcohol lamp until completely dissolved, and then cooled to room temperature to form a hydrogel.

\section{Circular Dichroism (CD) Spectrum}

A CD spectrophotometer (BioLogic, MOS-450, France) system was used to record the circular dichroism spectrum. Test wavelengths ranged from $185 \mathrm{~nm}$ to $280 \mathrm{~nm}$. The self-assembled IGF-1C hydrogel was added to a $0.1 \mathrm{~cm}$ quartz spectrophotometer cell $(20-\mathrm{C} / \mathrm{Q} / 0.1)$. The final spectrogram was obtained after subtracting the PBS solvent background.

\section{Surface Plasmon Resonance (SPR) Analysis}

SPR was performed using a BIAcore 2000 system (Biacore Inc., Piscataway, NJ). ${ }^{11,33}$ Soluble recombinant human IGF-1 receptor (rhIGF-1R) was immobilized on research grade CM5 chips (Biacore Inc., Piscataway, NJ) before chip activation with 1-ethyl-3(3-dimethyl aminopropyl) carbodiimide (EDC) and N-hydroxysuccinimide (NHS). To obtain kinetic data, different concentrations of analytes in HBSEP buffer (0.01 M HEPES, $\mathrm{pH}$ 7.4, 0.15 $\mathrm{M} \mathrm{NaCl}, 3 \mathrm{mM}$ EDTA, $0.005 \%$ Tween $20 \mathrm{v} / \mathrm{v}$ ) were injected over the sensor chip at a flow rate of $50 \mathrm{~mL} /$ min. Peptide binding was measured in resonance units (RUs). At the end of each sample injection (120 s), HBSEP buffer was passed over the sensor surface to monitor the dissociation phase. The concentrations of self-assembled IGF-1C ranged from $1.56 \mathrm{nM}$ to $200 \mathrm{nM}$. Reference responses of blank flow cells were subtracted from those of rhIGF-1R flow cells for each analyte injection, and the dissociation rate constant $(\mathrm{Kd})$ for each interaction was determined by globally fitting the experimental data to a 1:1 interaction model with BIA evaluation software (Biacore Inc., Piscataway, NJ).

\section{Transmission Electron Microscopy (TEM)}

A negative staining technique was used to observe the microstructure of the hydrogel. Ten microliters of hydrogel was added to a 400 objective copper network with a pipette. The gel was washed twice with water and dried with absorbent paper. The thin layer of hydrogel was stained with uranium acetate for $1 \mathrm{~min}$ and dried with absorbent paper and finally imaged by TEM (FEI, Talos F200X G2, USA).

\section{Rheological Test}

Rheological properties were tested by rheometer system (TA, AR 2000ex, USA). One milliliter of assembled IGF$1 \mathrm{C}$ hydrogel at a concentration of $0.5 \mathrm{wt} \%$ was loaded onto a $40 \mathrm{~mm}$ plate to test mechanical property dynamics along with the scanning frequency, temperature and strain. Briefly, the clearance was set to $500 \mu \mathrm{m}$, the temperature was set to $37^{\circ} \mathrm{C}$, the scanning frequency was set to $0.1 \mathrm{rad} / \mathrm{s}$ to $100 \mathrm{rad} / \mathrm{s}$, and the strain was set to $1 \%$.

\section{Cell Culture and Labeling}

HP-MSCs were purchased from H\&B (Beijing, China). Human umbilical vein endothelial cells (HUVECs) were purchased from ATCC (Manassas, VA). HP-MSCs were cultured with DMEM/F12 containing 8\% FBS, 1\% penicillin-streptomycin solution and 1\% MEM-NEAA solution. HUVECs were cultured with EGM-2 medium. To track the transplanted cell fate in vivo and in vitro, hPMSCs were transduced with a self-inactivating lentiviral 
vector carrying a ubiquitin promoter driving firefly luciferase (Fluc), thus, Fluc labeled hP-MSCs were obtained.34-36

\section{Cell Viability Assay}

To assess the effect of $\beta$-IGF-1C hydrogel on hP-MSCs, a Cell Counting Kit 8 (CCK-8) assay was performed. In brief, hP-MSCs were seeded in 96-well plates $\left(2 \times 10^{3} /\right.$ well $)$ and treated with different concentrations of the $\beta$-IGF-1C hydrogel $(0-200 \mathrm{nM})$ for 48 hours. Then, $10 \mu \mathrm{L}$ CCK-8 solution was added to each well and incubated for 3 hours at $37^{\circ} \mathrm{C}$. The optical density (OD) values at $450 \mathrm{~nm}$ were read with a microplate reader (Promega, WI, USA). Bioluminescent imaging (BLI) of hP-MSCs in vitro was performed with an In vivo Imaging System (IVIS) Lumina (Xenogen Corporation, Hopkinton, MA) as described previously ${ }^{37}$.

\section{Tube-Formation Assay}

To examine the proangiogenic effects of the $\beta$-IGF-1C hydrogel, a tube-formation assay was performed. ${ }^{38} \mathrm{hP}$ MSCs were cultured with $\beta$-IGF-1C (at a final concentration of $100 \mathrm{nM}$ ) for 48 hours and the supernatants were collected. Tube formation of HUVECs was assessed by seeding cells with the supernatants in 48 -well plates coated with Matrigel and incubating them at $37^{\circ} \mathrm{C}$ for 6 hours. The tube formation was quantitatively analyzed with Image J software (National Institute of Health, USA).

\section{BLI Analysis}

To monitor the Fluc signals from labeled hP-MSCs and the proangiogenic effect of the $\beta$-IGF-1C hydrogel in transgenic Vegfr2-Fluc-KI mice in real time, BLI was performed using an IVIS Lumina. ${ }^{36}$ D-Luciferin $(150 \mathrm{mg} /$ $\mathrm{kg}$; Biosynth International, Naperville, IL) was intraperitoneally administered to each mouse and the Fluc signal was captured from $1 \mathrm{~s}$ to $10 \mathrm{~min}$.

\section{Animal Surgery and Cell-Based Therapy}

Transgenic mice (Vegfr2-Fluc-KI) that were 8-12 weeks-old were raised in a specific pathogen-free (SPF) animal area at the Animal Facility of Nankai University. The protocol was approved by the Animal Ethics Committee of the Nankai University according to the Animal Care and Use Committee Guidelines, which conformed to the Guide for the Care and Use of Laboratory Animals published by the NIH. The ischemia/reperfusion ( $\mathrm{I} / \mathrm{R})$ induced acute kidney injury (AKI) model was set up as previously described. ${ }^{17}$ Briefly, animals were anesthetized by intraperitoneal injection of phenobarbitol sodium ( $150 \mathrm{mg} / \mathrm{kg}$ ). Following a dorsal longitudinal incision, right kidney was exposed and renal pedicle was clamped with a nontraumatic microvascular clamp for $40 \mathrm{~min}$. Reperfusion was confirmed visually before the incision was closed. After 15 minutes of reperfusion, $2 \times 10^{6} \mathrm{hP}$ MSCs stably expressing Fluc were intrarenally injected into 2 random sites of right kidney cortex at $40 \mu \mathrm{L}$ total volume suspended in PBS, or $\beta$-IGF-1C hydrogel respectively. PBS injection were served as control. Sham-operated animals were subjected to the same surgical procedure without renal ischemia or cell/hydrogel transplantation.

\section{Histological Assay and Scoring}

To investigate the therapeutic effects of the $\beta$-IGF-1C hydrogel, mice were sacrificed on days 3 and 28. Kidney samples were collected and fixed. Paraffin sections $(5 \mu \mathrm{m})$ were stained with periodic acid-Schiff (PAS), and Masson's trichrome. Immunofluorescence staining was carried out at the indicated time points.

\section{Renal Function Analysis}

To assess the renal function, mice underwent unilateral (right) 40-minute I/R plus contralateral nephrectomy. At different time points after injury, blood samples were harvested and serum was collected for assessment of blood urea nitrogen (BUN) and creatinine levels using biochemical automatic analyzer (Vitalab Selectra E, Netherlands).

\section{Western Blotting Analysis}

The hP-MSCs were lysed with RIPA buffer. The protein concentrations were quantified by BCA protein assay Kit. The total proteins $(20 \mu \mathrm{g})$ were separated by $12 \%$ sodium dodecyl sulphate polyacrylamide gel electrophoresis (SDS-PAGE) and transferred to polyvinylidene fluoride (PVDF) (Millipore, Watford, UK) membranes. The membranes were blocked with 5\% skim milk for 2 hours, then incubated overnight in the primary antibodies at $4^{\circ} \mathrm{C}$, then incubated for 2 hours at room temperature with the appropriate secondary antibodies at the next day. Signals of Western blot was detected by Luminata crescendo western horseradish peroxidase substrate (Millipore).

\section{Statistical Analysis}

Statistical analysis was performed using SPSS software 24 (IBM), and all data were expressed as the mean \pm Standard Error of Mean (SEM). Student's $t$ test and one-way ANOVA were carried out for comparison between two independent groups and multiple groups respectively. The correlation 
between Fluc-hP-MSC numbers and Fluc signal intensity was tested by Pearson correlation (two-tailed). Differences were considered significant at $P$ values $<0.05$.

\section{Results}

\section{Synthesis and Characterization of a Self- Assembled IGF-IC Hydrogel}

To resist natural enzyme degradation, D-form self-assembling peptide nanofiber scaffolds have been used for cell culture and tissue regeneration. ${ }^{2,30,39,40}$ IGF-1 mediates cell growth and differentiation and has been widely used in tissue engineering and regenerative medicine. ${ }^{17,41}$ Therefore, the design of biomimetic scaffolds with bioactivity-mimicking peptide IGF-1C and D-form self-assembling peptide motif Nap-FFG was developed. Here we introduced D-form amino acids into the self-assembly motif Nap-FFG and covalently attached Nap- ${ }^{\mathrm{D}} \mathrm{F}^{\mathrm{D}} \mathrm{FG}$ to the N-terminus of IGF-1C to obtain a self-assembling IGF-1C hydrogel (Figure 1A). Nuclear magnetic resonance ${ }^{1} \mathrm{H}-\mathrm{NMR}$ and mass spectrometry (MS) analysis confirmed the successful synthesis (Supplemental Figure 1) with the exact molecular weight (Supplemental Figure 2).

Synthetic hydrogels originating from supramolecular self-assembly of building blocks endow precise control over composition and biophysical properties. Rheological analysis was carried out to examine the gelation properties of the self-assembling IGF-1C hydrogel. Our results revealed the viscoelasticity of this hydrogel with respect to a change in frequency (Figure 1B). With increasing frequency from 0.1 to $100(\mathrm{rad} / \mathrm{s})$, the storage modulus G' always exceeded the loss modulus G', suggesting that the self-assembling peptide nanofibers adopted a stable hydrogel. Consistent results were obtained with temperature (Supplemental Figure 3A) and stress changes (Supplemental Figure 3B). We next conducted a CD spectrum analysis to examine the putative superior structure of this hydrogel. Our data revealed that this self-assembling IGF-1C hydrogel exhibited a positive peak at $195 \mathrm{~nm}$ and a negative band at $219 \mathrm{~nm}$, which indicated a classical $\beta$ sheet formation. The unassembled IGF-1C solution showed no distinct peak, suggesting it adopts a random coil structure (Figure 1C). Optical images further confirmed the hydrogel property (Figure 1D).

SPR spectroscopy analysis was carried out to test the binding affinity of this self-assembling $\beta$-sheet IGF-1C peptide ( $\beta$-IGF-1C) hydrogel with the recombinant human IGF-1 receptor (rhIGF-1R). Our results indicated that this
$\beta$-IGF-1C hydrogel exhibited an equilibrium dissociation constant $(\mathrm{Kd})$ value of $8.2 \mathrm{nM}$ for the rhIGF-1R, while the L-form amino acids self-assembling $\beta$-sheet IGF-1C peptide hydrogel revealed a higher $\mathrm{K}_{\mathrm{D}}$ value of $11.5 \mathrm{nM}$ (Figure 1E). These findings indicated that this D-form self-assembling peptide hydrogel has a superior binding affinity with IGF-1R and provides powerful evidence for its potential bioactivity. To explore the ultrastructure of the $\beta$-IGF-1C hydrogel, TEM was performed and an entangled nanofiber structure was observed (Figure 1F).

\section{Biocompatibility of the $\beta$-IGF-IC Hydrogel}

A CCK-8 assay was performed to evaluate the proproliferation activity of the $\beta$-IGF-1C hydrogel. The results indicated that the optimal concentration of the $\beta$-IGF-1C hydrogel was $100 \mathrm{nM}$ (Figure 2A). We then cultured hPMSCs with the $\beta$-IGF-1C hydrogel (100 nM), and these cells were labeled with firefly luciferase (Fluc). BLI showed that hP-MSCs expanded rapidly when cultured on $\beta$-IGF-1C hydrogel-coated plates (Figure 2B and C). Consistent effects of $\beta$-IGF-1C hydrogel on HUVECs were also observed (Supplemental Figure 4).

To investigate the proangiogenic effects of the $\beta$-IGF1C hydrogel, HUVECs were pretreated with supernatant from hP-MSCs cultured with the $\beta$-IGF-1C hydrogel. A tube-formation assay revealed that the number of nodes was increased in the group of HUVECs primed with the supernatant (Figure 2D and E). To examine the cell protective effects of the $\beta$-IGF-1C hydrogel, HUVECs were treated with hydrogen peroxide $\left(\mathrm{H}_{2} \mathrm{O}_{2}\right)(200 \mu \mathrm{M})$ for 2 hours, and cell survival was determined by live/dead staining. Our data demonstrated that the $\beta$-IGF-1C hydrogel ameliorated cell apoptosis induced by $\mathrm{H}_{2} \mathrm{O}_{2}$ (Supplemental Figure 5).

\section{Improvement of Cell Engraftment}

To track the fate of transplanted cells in vivo, hP-MSCs were transduced with a self-inactivating lentiviral vector carrying an ubiquitin promoter driving Fluc reporter gene. Optical imaging revealed a typical morphology of hPMSCs (Figure 3A). Upon culturing in 24-well plates, we also observed a strong correlation $\left(\mathrm{R}^{2}=0.99\right)$ between Fluc activity and cell numbers ex vivo (Figure 3B and C), which indicated the possibility of assessing cell retention in vivo by analyzing the Fluc signal intensity. To explore the cell enhancement of the $\beta$-IGF-1C hydrogel, we 
A

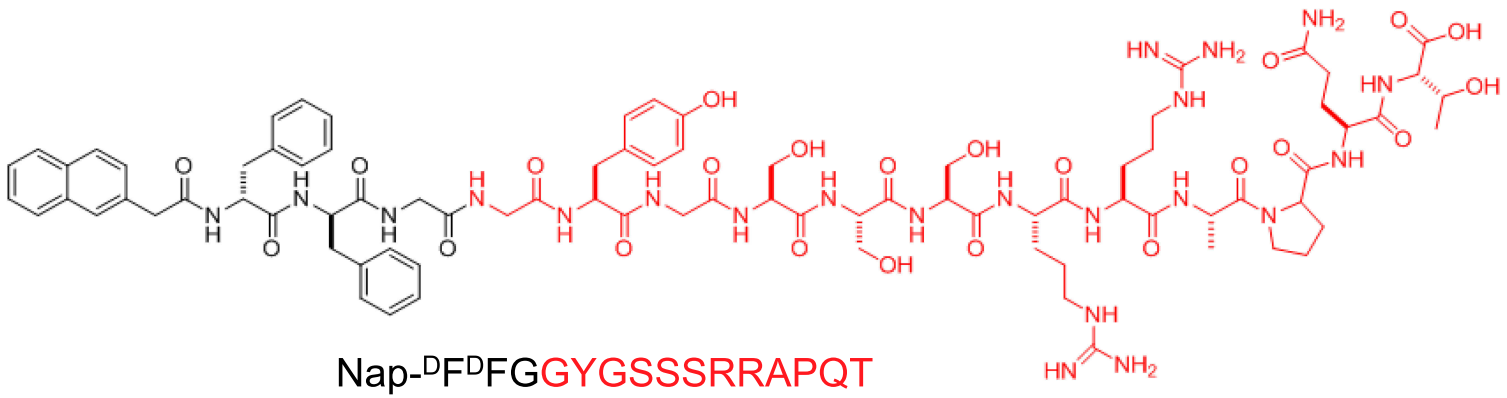

B

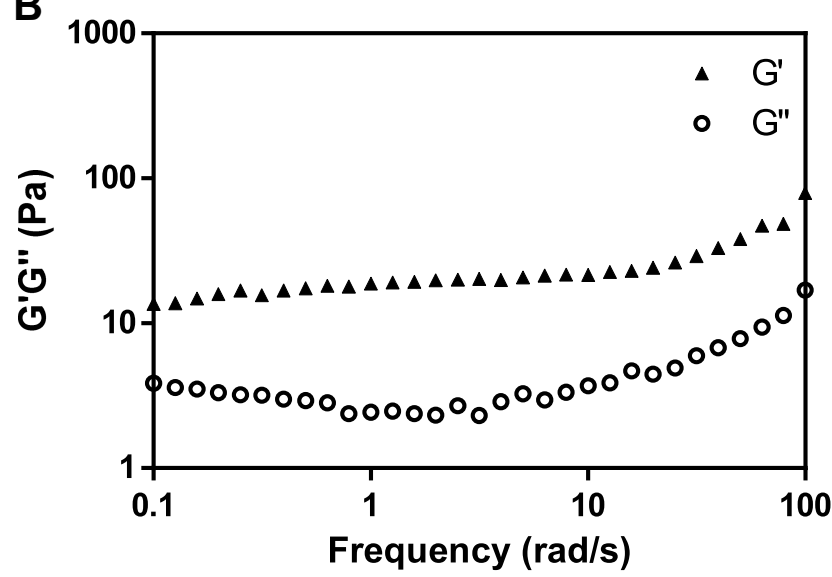

D

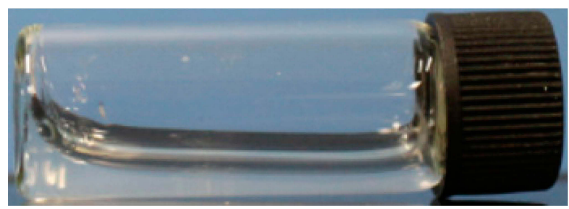

Unassembled

E

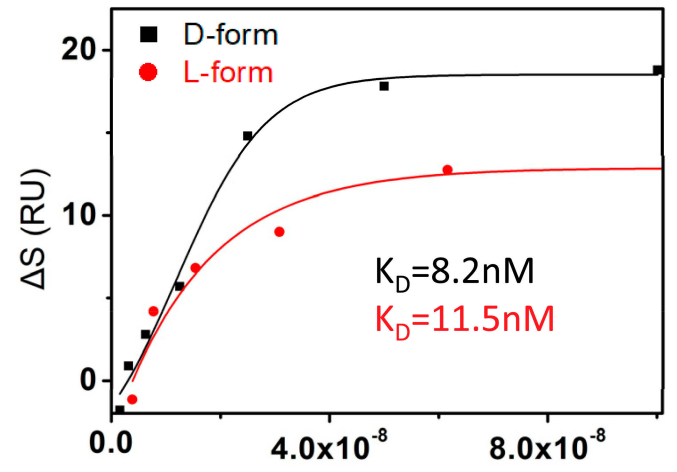

C

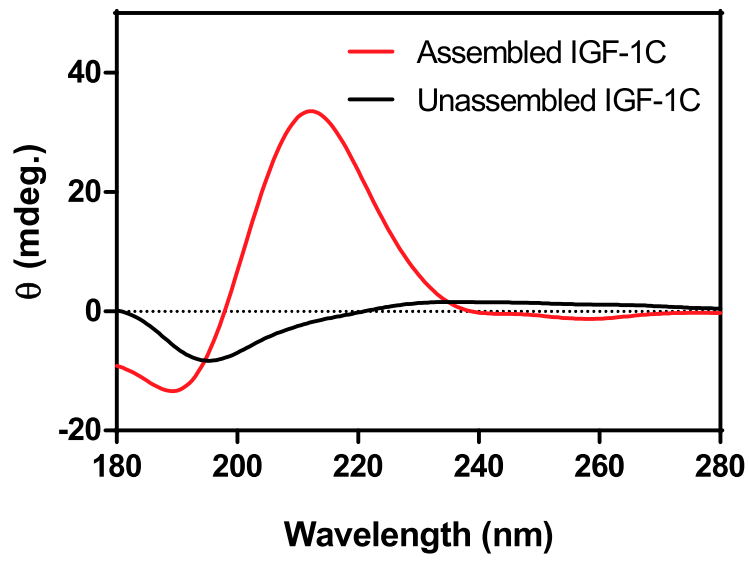

Concentration(M)

Figure I Synthesis and characteristics of a self-assembling IGF-IC hydrogel. (A) Chemical structure of the self-assembling IGF-IC hydrogel. (B) Frequency-dependent rheological analysis of self-assembling IGF-IC hydrogel. (C) Circular dichroism (CD) spectra of the self-assembling IGF-IC hydrogel compared with that of an unassembled one. (D) Optical images of unassembled IGF-IC (solution) versus assembled IGF-IC (hydrogel) in the phosphate buffered solution (PBS) buffer solvent (pH=7.4). (E) Surface plasmon resonance (SPR) response units of the $\beta$-IGF-IC hydrogel with recombinant human IGF-I receptor. (F) Transmission electron microscopy (TEM) image of the ultrastructure of $\beta$-IGF-IC hydrogel. Scale bar $=500 \mathrm{~nm}$. 

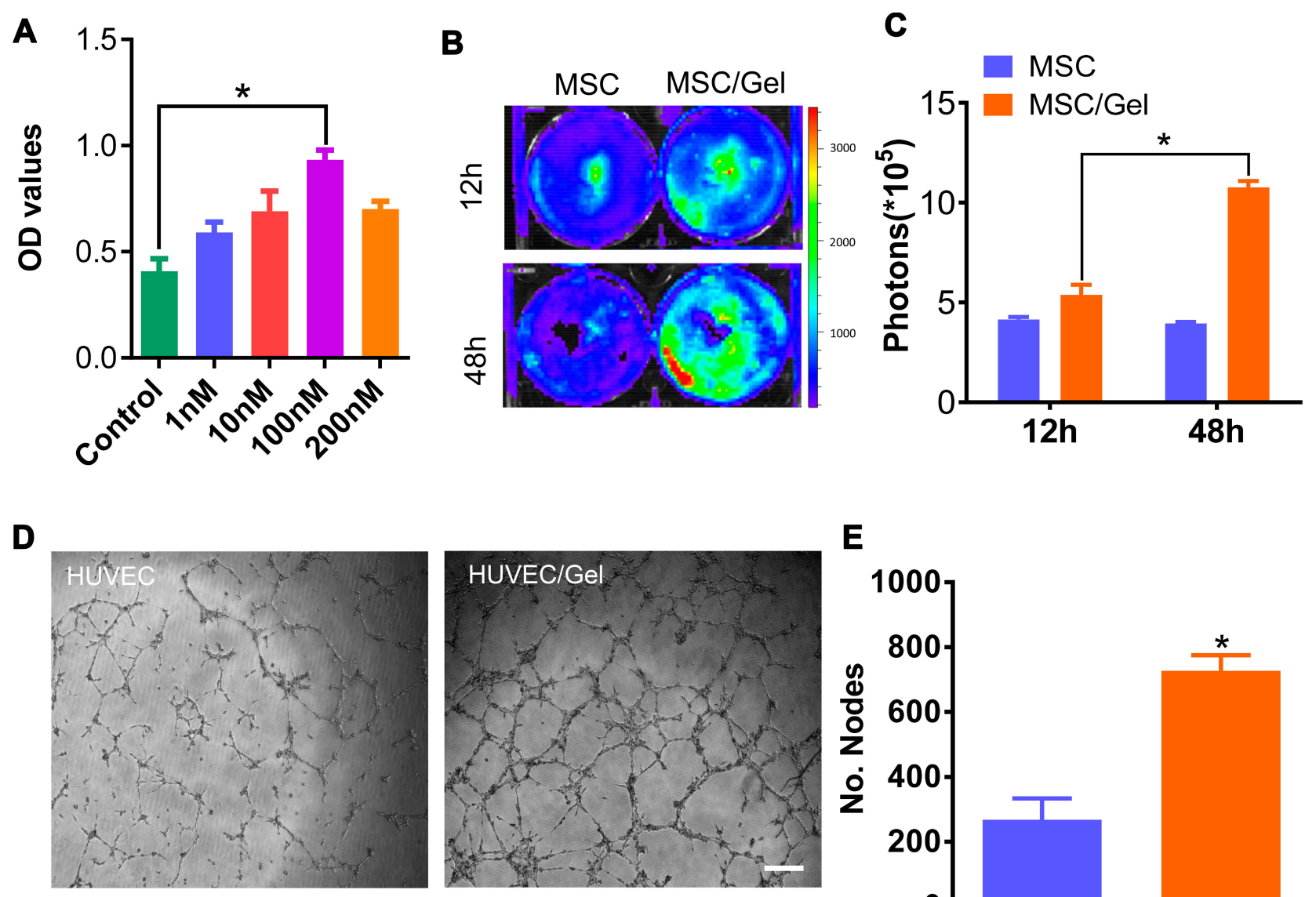

\section{E}

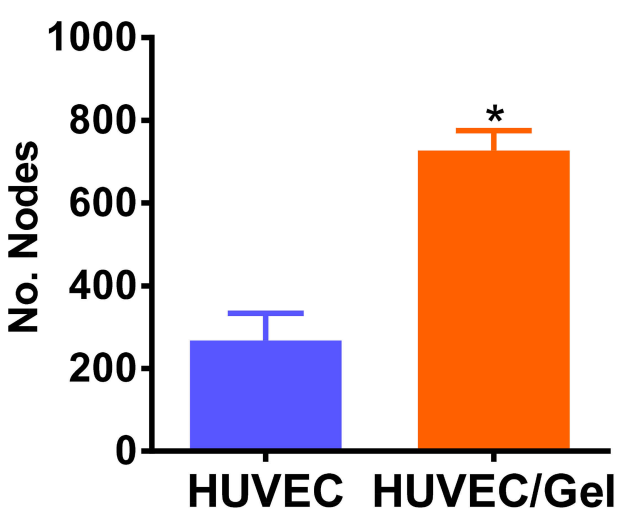

Figure 2 Biocompatibility of the $\beta$-IGF-IC hydrogel. (A) Cell Counting Kit 8 (CCK-8) assay demonstrating the optical density (OD) values of human placenta-derived mesenchymal stem cells (hP-MSCs) in the presence of the $\beta$-IGF-IC hydrogel at diverse concentrations $(0-200 \mathrm{nM})$. $* P<0.05$ versus Control. (B) Bioluminescence living imaging (BLI) image of Fluc-hP-MSCs cultured with $\beta$-IGF-IC hydrogel (I00 nM) at different time points (I2 and 48 hours) (C) Quantitative analysis of firefly luciferin (Fluc) signal intensity. Data are expressed as the means \pm SEM. $* P<0.05$ versus Control. (D) Optical images of tube formation of Human umbilical vein endothelial cells (HUVECs). Scale bar $=100 \mu \mathrm{m}$. (E) Quantitative evaluation of nodes by using Image J software. Data are expressed as the mean \pm SEM. $* P<0.05$ versus Control.

transplanted Fluc labeled hP-MSCs with the $\beta$-IGF-1C hydrogel into a mouse model of AKI. Our BLI data revealed a robust signal at day 1 after intrarenal delivery, suggesting successful cell transplantation. Serial BLI demonstrated that $\beta$-IGF-1C hydrogel significantly improved hP-MSC retention (Figure 3D and E). These results indicated that the $\beta$-IGF-1C hydrogel provides a promising scaffold for transplanted stem cells.

\section{Improvement of Kidney Function}

To investigate the renal-protective effects, histological examinations were carried out. Hematoxylin and eosin (H\&E), periodic acid-Schiff (PAS) staining at day 3 (Figure 4A) and acute tubular necrosis (ATN) scoring (Figure 4B and C) revealed functional recovery with $\mathrm{hP}$ MSC or $\beta$-IGF-1C hydrogel treatment, and further amelioration was achieved by the cotransplantation of hP-MSCs and $\beta-\mathrm{IGF}-1 \mathrm{C}$. AKI is characterized by deterioration of renal function with increased serum creatinine $(\mathrm{SCr})$ and high BUN. To investigate renal function recovery after AKI, serum was harvested from mice submitted to the bilateral AKI model. Significant increases in BUN and $\mathrm{SCr}$ were observed in the PBS group, which were alleviated to a certain degree in the $\beta$-IGF-1C hydrogel and hP-MSC groups. Cotransplantation of the $\beta$-IGF-1C hydrogel and hP-MSCs demonstrated best functional improvement (Figure 4D and E).

To investigate whether the $\beta$-IGF-1C hydrogel and hP-MSC cotransplantation could benefit endogenous regeneration in AKI model, we conducted the immunofluorescence for KIM-1 and Ki-67, injury and regeneration markers respectively. Our data demonstrated that KIM-1 expression decreased significantly, while Ki-67 expression increased robustly with hP-MSCs or $\beta$-IGF-1C hydrogel 
A

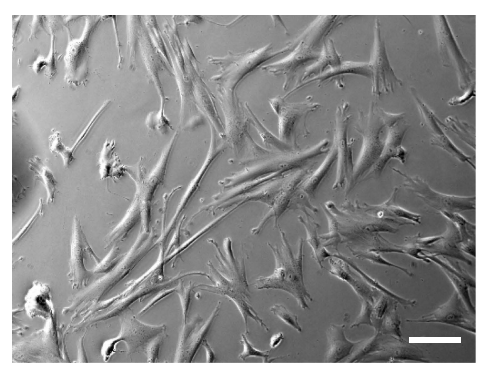

B

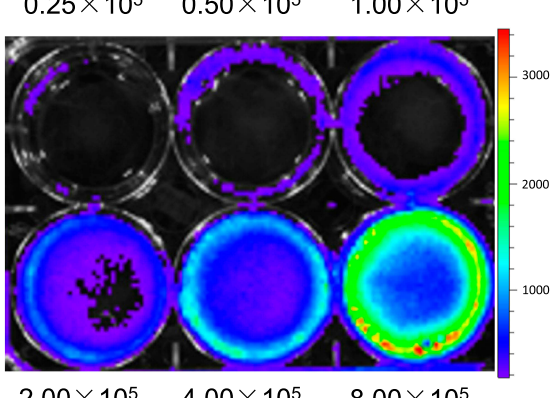

$2.00 \times 10^{5}$

$8.00 \times 10^{5}$
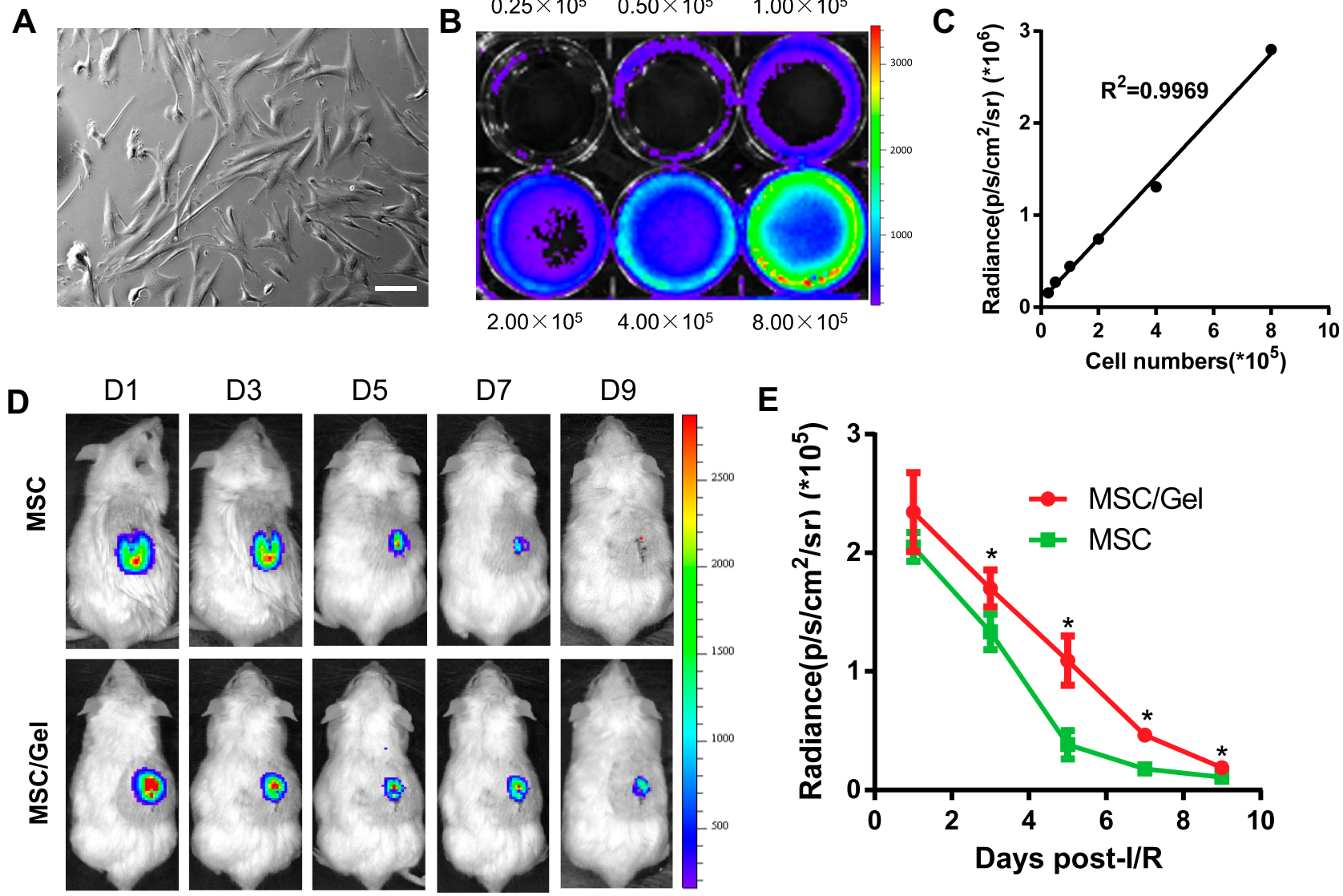
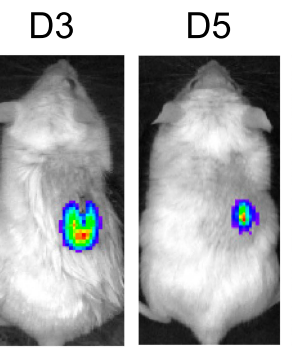

D7
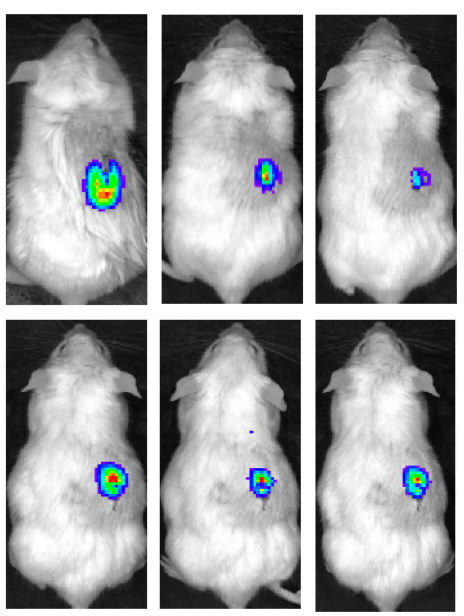

D9

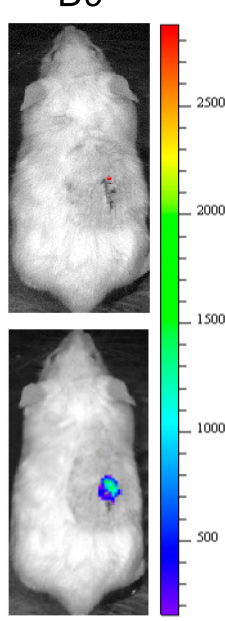

E

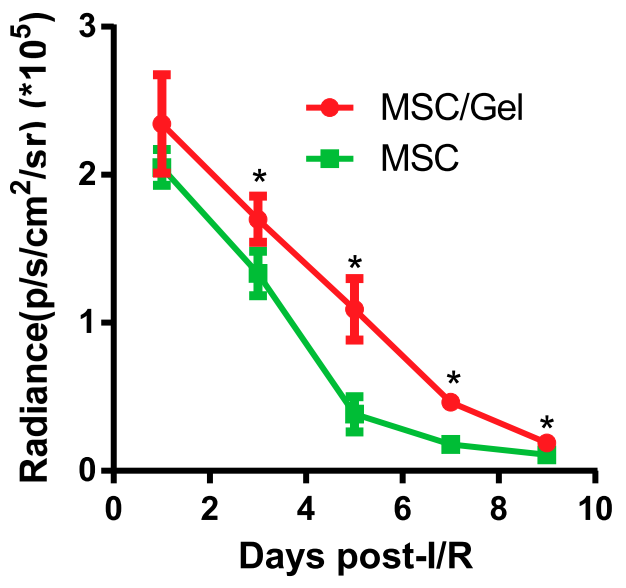

Figure 3 Improvement of cell engraftment. (A) Bright field image of human placenta-derived mesenchymal stem cells (hP-MSCs). Scale bar=50 $\mu$ m. (B) Firefly luciferin (Fluc) activities of labeled hP-MSCs. The experiment was performed in triplicate. (C) Correlation between cell numbers and Fluc expression. (D) Longitudinal tracking of labeled cell fate over a 9-day period in a murine acute kidney injure (AKI) model via bioluminescence living imaging (BLI). (E) Quantification of the time-dependent Fluc signal dynamics, as an equivalent of cell retention. Data are expressed as the mean \pm SEMs, $* P<0.05$ versus hP-MSCs.

treatment, and these trends were strengthened by cotransplantation of hP-MSCs and the $\beta$-IGF-1C hydrogel (Figure 4F). Quantitative analysis confirmed this tendency (Figure $4 \mathrm{G}$ and $\mathrm{H}$ ).

IGF-1 receptor is a transmembrane receptor tyrosine kinase that is widely expressed in many cell lines and cell types within fetal and postnatal tissues. ${ }^{42}$ Receptor autophosphorylation follows binding of IGF-1 ligand, and subsequently activate the downstream signal pathway. We analyzed the phosphorylated IGF-1R in hP-MSCs with or without $\beta$-IGF-1C treatment, and our data revealed that $\beta$ IGF-1C treatment could further increase the receptor phosphorylation in hP-MSCs, which responds to the enhanced therapeutic efficiency of the hP-MSCs (Figure 4I).

\section{Attenuated Renal Fibrosis}

With excessive accumulation and deposition of ECM, renal fibrosis is the common final pathway to renal injuries that ultimately impairs kidney function and consequently leads to end-stage kidney disease (ESKD). ${ }^{17}$ To ameliorate renal fibrosis, many experimental studies have been conducted to explore new strategies to blunt the processes of renal fibrogenesis. Masson trichrome staining and collagen IV immunofluorescence revealed a significant reduction of collagen fibers deposition in the hP-MSCs and $\beta$-IGF-1C hydrogel cotransplantation group (Figure 5A). Quantitative analysis demonstrated that the deposition of collagen fibers was alleviated in both the $\beta$-IGF-1C hydrogel and hP-MSC groups, and the best recovery was observed in the cotransplantation group (Figure 5B and C).

\section{Proangiogenic Effects of $\beta$-IGF-IC Hydrogel}

Regeneration of the kidney requires sufficient blood supply and a strategy to promote angiogenesis will contribute to the functional recovery of kidney injury. To investigate whether the $\beta$-IGF-1C hydrogel favors angiogenesis, transgenic mice (Vegfr2-Fluc-KI) ${ }^{43}$ were applied as a reporter tool for dynamic tracking of angiogenesis in AKI model. 
A
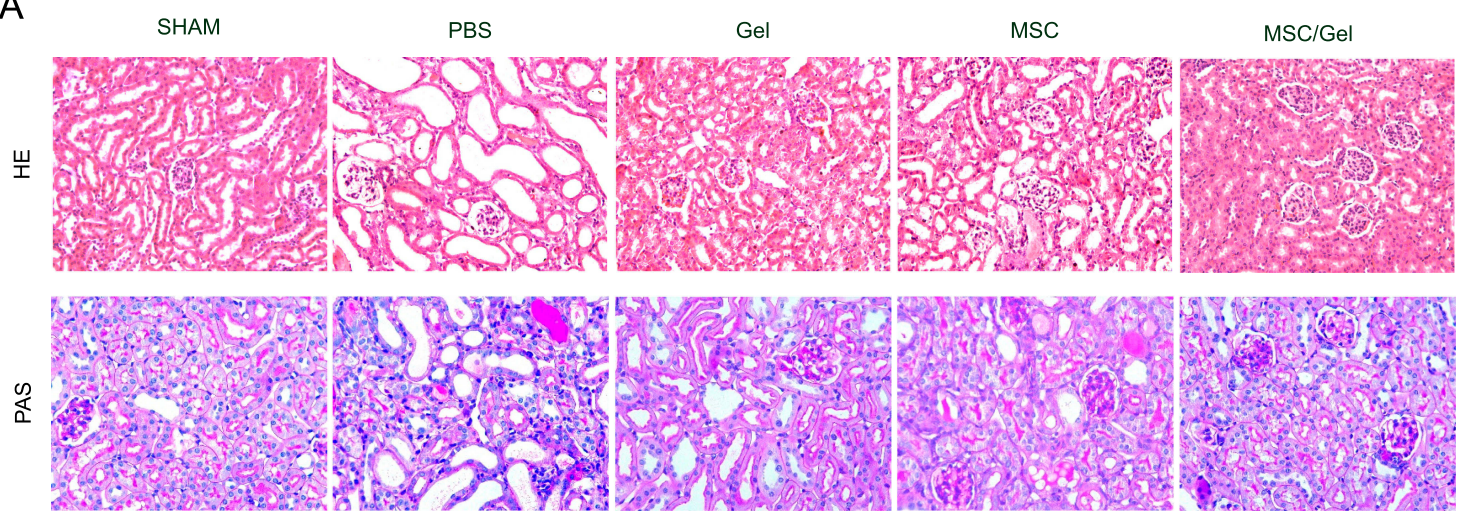

B

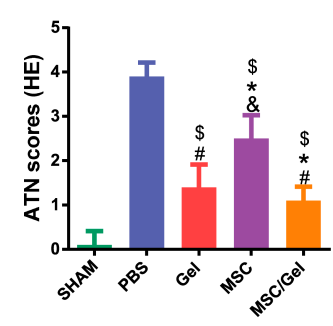

F
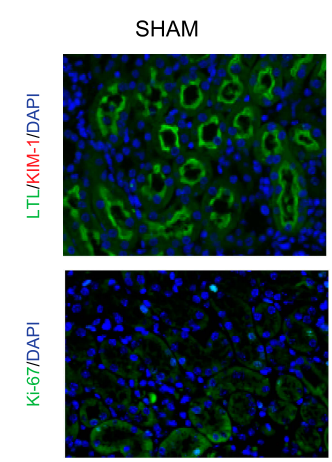

G

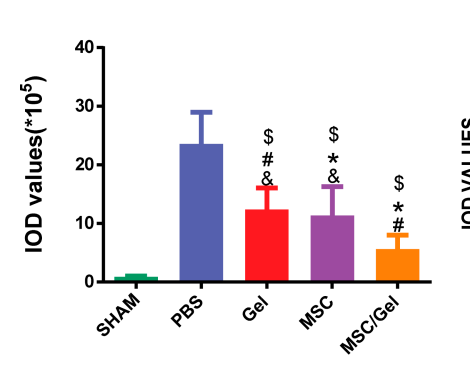

$\mathrm{H}$
C

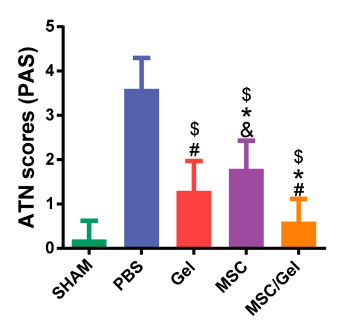

D

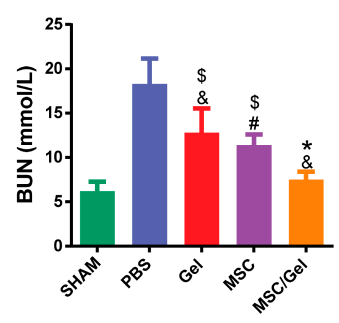

E

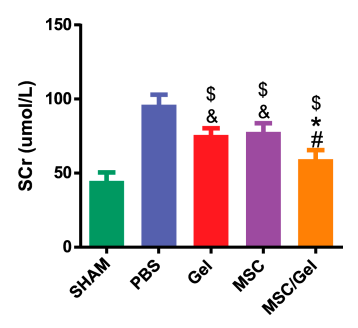

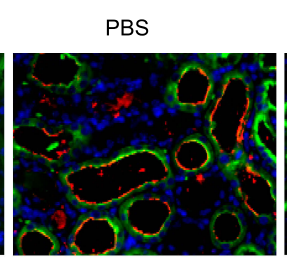
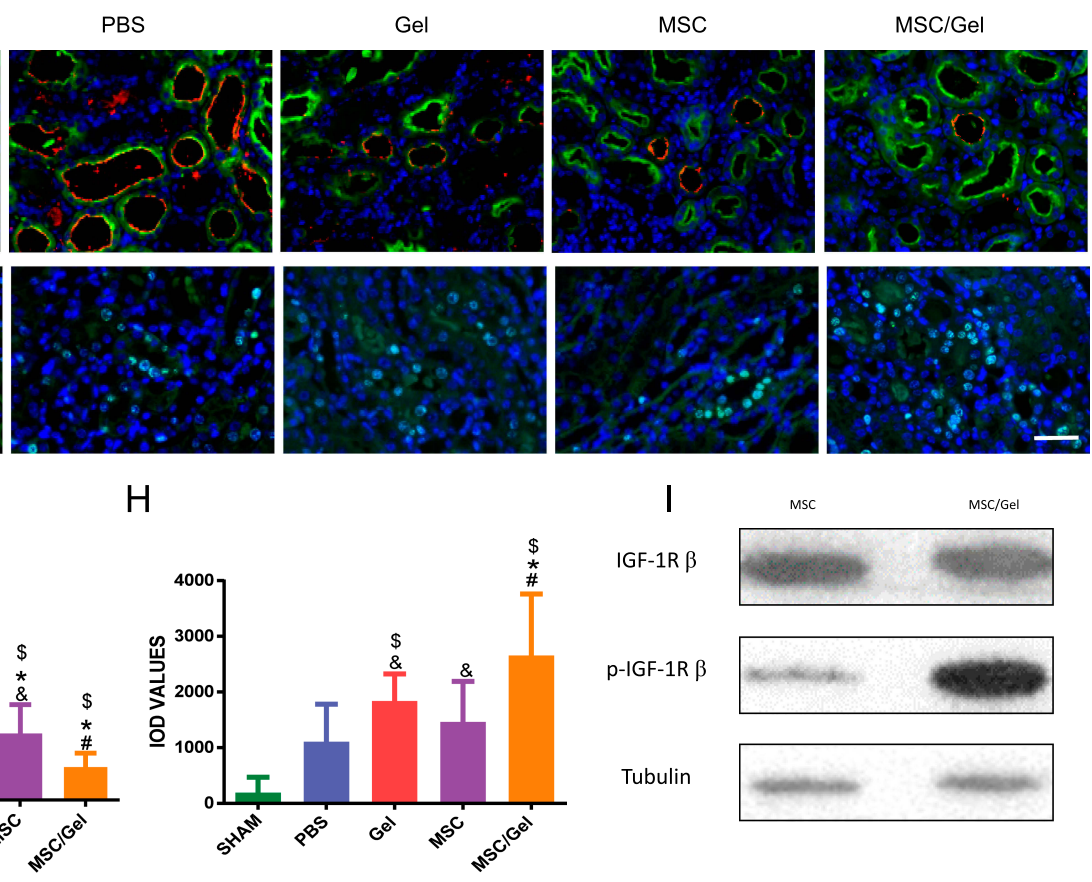

IGF-1R $\beta$

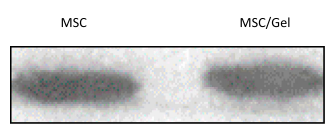

p-IGF-1R $\beta$

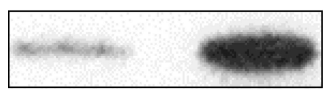

Tubulin

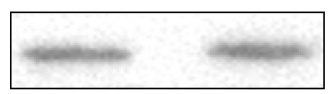

Figure 4 Ameliorated renal repair post-injury. (A) Histological assay by Hematoxylin and Eosin (HE), periodic acid-Schiff (PAS) staining revealing the repair process at day 3 post-acute kidney injure (AKI). $n=5$ for each group. Scale bar $=50 \mu \mathrm{m}$. (B) HE and (C) PAS semiquantitative histological assessment via acute tubular necrosis (ATN) scoring. (D) Blood urea nitrogen (BUN) and (E) serum creatinine (SCr) levels as indicators of renal function were detected at day I post-injury. (F) Representative images of kidney injure molecular-I (KIM-I) and Ki-67 immunofluorescence staining on day 3. Scale bar=50 $\mu \mathrm{m}$. Lotus Tetragonolobus Lectin (LTL) was stained as a marker of proximal tubular epithelial cells, while nuclei were counter stained with 4, 6-diamino-2-phenyl indole (DAPI). Scale bar=50 $\mu \mathrm{m}$. (G) Integrated optical density (IOD) values for KIM-I and (H) Ki-67. All data mentioned above are expressed as the mean \pm SEM. ${ }^{\$} P<0.05$ versus phosphate buffered solution (PBS); ${ }^{\#} P<0.05$ versus human placenta-derived mesenchymal stem cells (hP-MSCs); ${ }^{* P}<0.05$ versus $\beta$-IGF-IC; ${ }^{\circledR} P<0.05$ versus $\beta$-IGF-IC+hP-MSCs. (I) Western blot analysis of phosphorylated IGF-I receptor of hP-MSCs with or without $\beta$-IGF-IC treatment. 


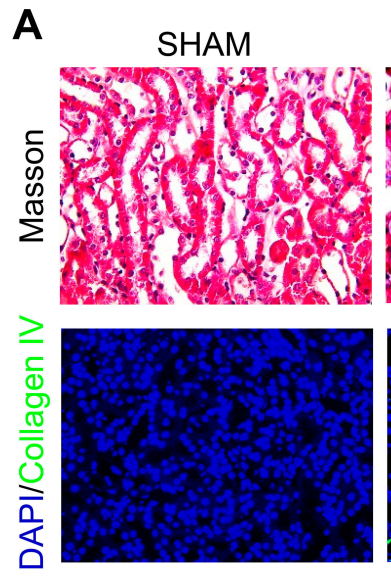

B
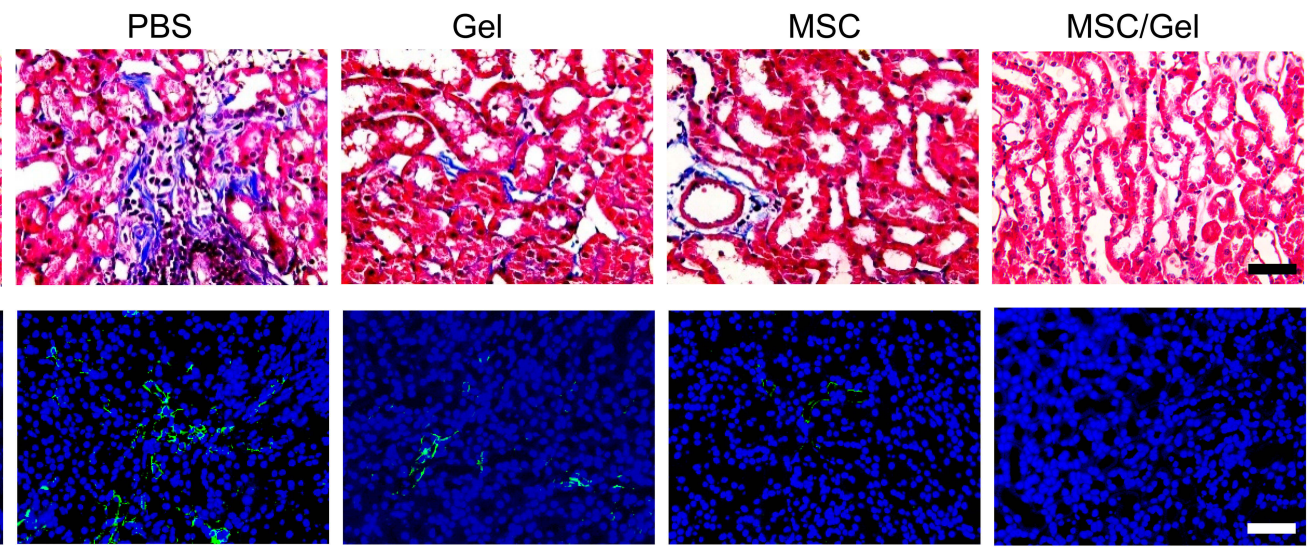

C
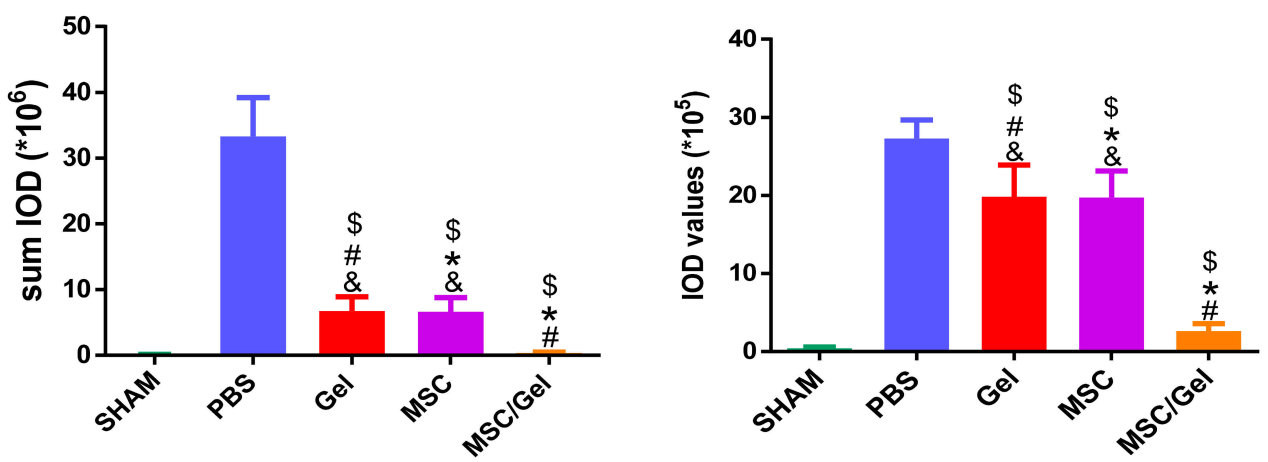

Figure 5 Attenuated renal fibrosis. (A) Representative images of kidney sections stained for Masson trichrome and immunofluorescence images of collagen IV at day 28 after acute kidney injure (AKI), while nuclei were counter stained with 4, 6-diamino-2-phenyl indole (DAPI). Scale bar=50 $\mu \mathrm{m}$ (upper panel) or, I00 $\mu \mathrm{m}$ (lower panel). (B) Semiquantitative analysis of Masson staining via Image J software. (C) Integrated optical density (IOD) values of collagen IV, as an equivalent to kidney fibrosis. Data mentioned above are expressed as the mean \pm SEM. ${ }^{\$} P<0.05$ versus phosphate buffered solution (PBS); ${ }^{\#} P<0.05$ versus human placenta-derived mesenchymal stem cells (hPMSCs); ${ }^{*} P<0.05$ versus $\beta$-IGF-IC; ${ }^{\circledR} P<0.05$ versus $\beta$-IGF-IC+hP-MSCs.

Longitudinal BLI assays were performed on days $0,3,5$, 7, 10 and 14 after surgery. Our data showed that the strongest signals were observed in the $\beta$-IGF-1C hydrogel and hP-MSC cotransplantation group (Figure 6A). In addition to the sham group, BLI signals were detected in all groups, which indicated the activation of the VEGF/VEGFR2 pathway after injury and the $\beta$-IGF-1C hydrogel or hPMSCs could amplify this effect (Figure 6B). Further immunofluorescence staining of vWF, which behaves as a marker for angiogenesis, demonstrated that $\mathrm{vWF}$ expression was increased with $\beta$-IGF-1C hydrogel and hP-MSC treatment, which was consistent with the BLI data (Figure 6C).

\section{Discussion}

In the current study, we explored a de novo $\beta$-sheet supramolecular self-assembling peptide hydrogel, which combines the bioactive motif IGF-1C and the D-form assembly motif Nap- ${ }^{\mathrm{D}} \mathrm{F}^{\mathrm{D}} \mathrm{FG}$, together with hp-MSCs for AKI treatment. Our results revealed that this assembled hydrogel could further activate recombinant human IGF-1 receptor ex vivo, owing to its inherent $\beta$-sheet secondary structure, and benefit the engraftment and survival of transplanted hP$\mathrm{MSC}$ in vivo. Moreover, cotransplantation of the $\beta$-IGF-1C hydrogel and hP-MSCs contributed to endogenous regeneration post injury and boosted angiogenesis in a murine AKI model, leading to recovery of renal function.

MSCs have been shown to be effective for acute kidneys because of their versatile activities, such as paracrine, antioxidant or trans-differentiation ${ }^{17,44}$, which are ideal cell sources with hematopoietic supportive and immunomodulatory features. ${ }^{45}$ Nevertheless, the transplanted MSCs may suffer a significant apoptosis event called anoikis outside of ECM support. ${ }^{1}$ Cell-based therapy might be compromised due to low cell retention and poor niche establishment after transplantation. Therefore, fabricating novel biomaterials that simultaneously are competent ECM mimics and promote cell delivery is an urgent need. 
A

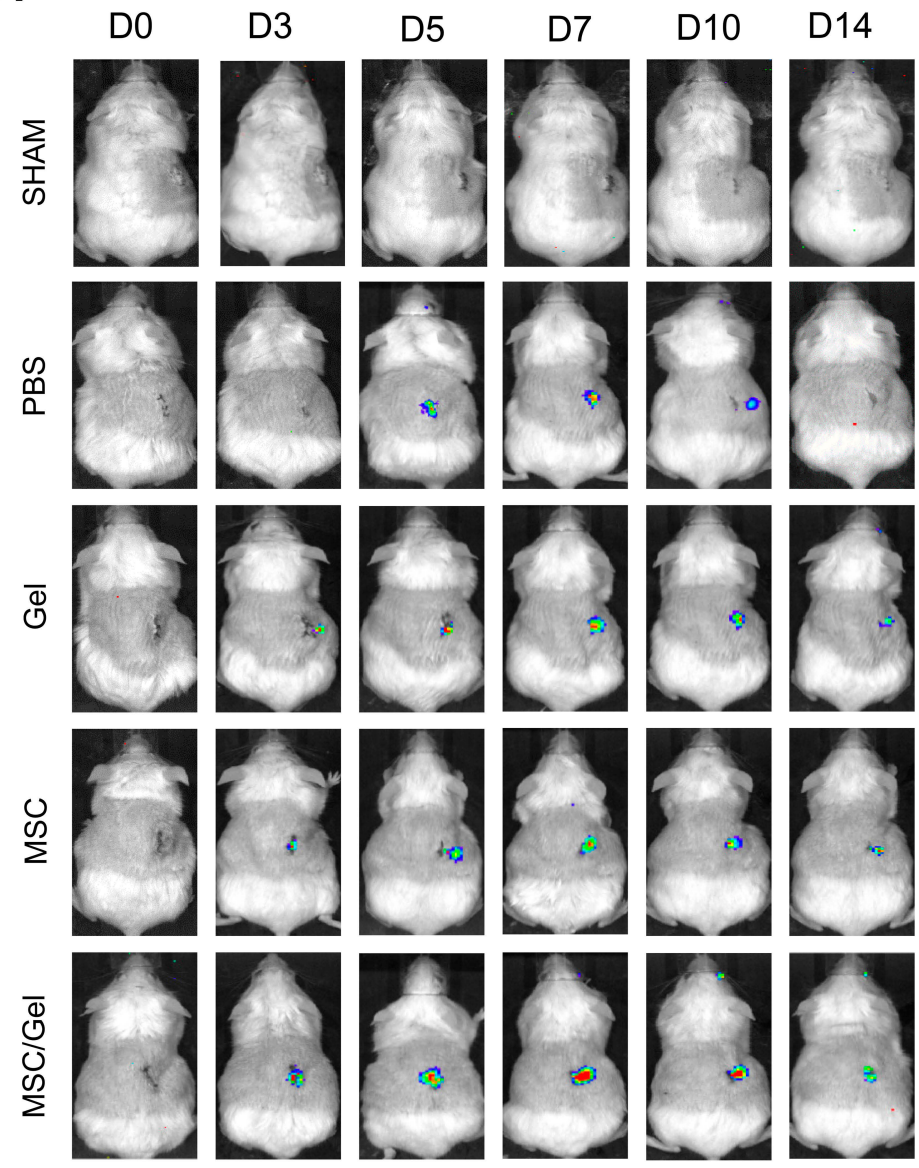

B

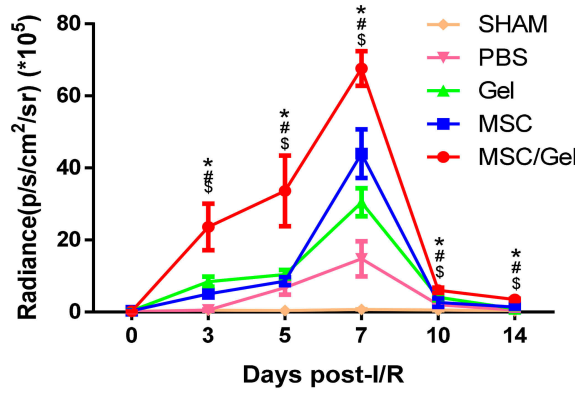

C
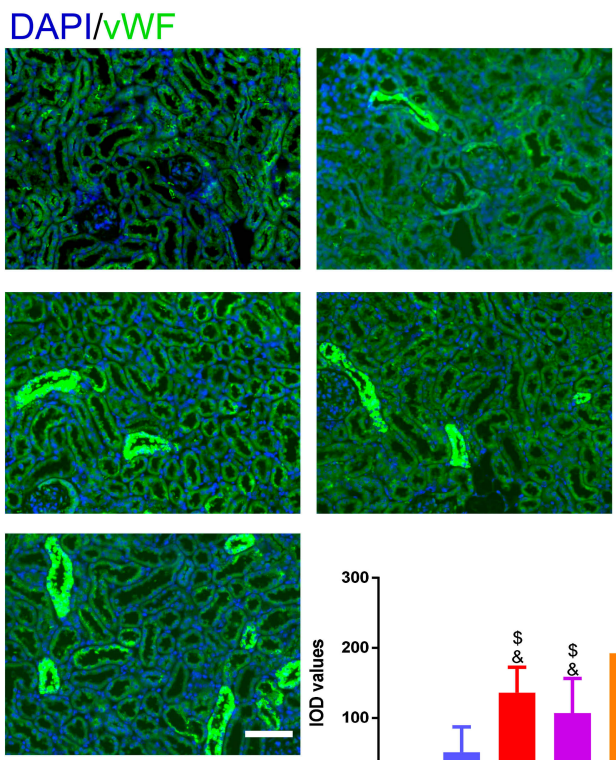

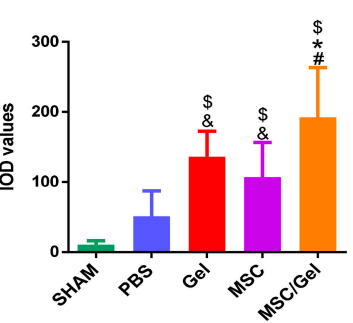

Figure 6 Accelerated angiogenesis in vivo. (A) In vivo bioluminescence living imaging (BLI) tracking of spatiotemporal kinetics of vegfr2 expression in murine acute kidney injure (AKI) model. (B) Quantification of vegfr2 expression by BLI. $n=5$ for each group. Data are expressed as the mean \pm SEM. ${ }^{\$} P<0.05$ versus phosphate buffered solution (PBS); ${ }^{\#} P<0.05$ versus human placenta-derived mesenchymal stem cells (hP-MSCs); ${ }^{*} P<0.05$ versus $\beta$-IGF-IC; ${ }^{\circledR} P<0.05$ versus $\beta$-IGF-IC+hP-MSCs. (C) Representative images and integrated optical density (IOD) values of von Willebrand factor (vWF) immunofluorescence staining of ischemic tissue at day 3 , while nuclei were counter stained with 4, 6-diamino-2-phenyl indole (DAPI). Scale bar $=100 \mu \mathrm{m}$.

Of the diverse biomaterials, synthesized supramolecular self-assembling peptide hydrogels can function as biomimetic materials to reproduce the nanofiber architecture and properties of ECM and thereby to serve as scaffolds for tissue engineering and regenerative medicine., , $^{2,46}$ Their properties can be readily tailored by changes at the sequence level, and homogeneity and standardization which are prerequisites for large volume production can be addressed by chemical synthesis and recombinant technologies. ${ }^{2,4,6}$ Biological self-assembly offers a versatile bottom up strategy to fabricate functional scaffolds imitating the complex hierarchy of natural tissues, which endows them with biomimetic ECM and cell delivery vehicle attributes. ${ }^{2,4,56}$ It is well accepted that superior structure results in enhanced function of peptides, and $\beta$ - sheet peptides are of great interest due to their amazing ability to reassemble. ${ }^{3,9}$ Our previous work has already shown that the $\beta$-sheet peptide hydrogels exhibited higher affinity for receptors than the unstructured counterparts ${ }^{11}$.

Chiral molecules are ubiquitous in nature. Likewise, all amino acids are either the D-form or the L-form. ${ }^{29}$ Selfassembling peptides can be obtained from both D-form and/or L-form amino acids. Amazingly, the enantiomerderived self-assembling peptides with identical amino acid residues showed significant differences in temperature and $\mathrm{pH}$ change resistance and even self-assembling ability. ${ }^{47}$ Accumulating studies have demonstrated that peptides made of only D-form amino acids are more resistant to protease degradation compared with their L-form counterparts, thus, self-assembling peptides with D-form amino 
acids are highly durable and seem to be a tangible option for improved scaffold stability. ${ }^{30,31,40}$

Hydrogel without functional motif is not sufficient to mimic all the properties of ECM in vivo due to the complex relationship between structure and biophysical functions. The morphology provides only an architectural support and a physiochemical signal but lacks an inherent biological motif., ${ }^{2,4}$ Therefore, hydrogels need to be further functionalized by introducing bioactive components of the ECM. Growth factors, as indispensable components of the ECM, are the appropriate components for this strategy. ${ }^{17,48}$ IGF-1 plays a crucial role in human childhood growth and continues to have anabolic effects in adults. ${ }^{17}$ Substantial advances in tissue engineering and regenerative medicine indicate the potential of using IGF-1 for wound healing and regeneration. ${ }^{18}$ The versatility of IGF-1 depends on its specific binding to its receptor, primarily IGF-1R. ${ }^{49}$ Previous work has demonstrated that IGF-1C might be largely responsible for the specific binding with IGF$1 \mathrm{R} .^{50}$ Thus, the IGF-1C mimic peptide could be utilized for hydrogel synthesis as surrogate of IGF-1.

The IGF-1C mimic peptide has gained increasing attention in the biomaterial field and tissue engineering. A chiston (CS) modified IGF-1C (CS-IGF-1C) hydrogel has been reported as a stem cell cargo for AKI therapy. ${ }^{17}$ However this hydrogel is actually a simple chemical mixture which combines the gelling motif CS and the functional motif IGF-1C. It in essence can be regarded as the unassembled IGF-1C, while the self-assembled IGF-1C exhibited a superior receptor affinity due to its inherent $\beta$-sheet secondary structure. ${ }^{11}$ Also we previously reported a $\beta$-sheet nanofiber with IGF-1C as its backbone for the ischemic hind limb therapy. In present study we introduced a D-form peptide to stabilize and optimize this hydrogel, and introduced it to the stem cell-based therapy. In addition, it is well acknowledged that IGF-1 ligand binds to IGF-1 receptor via its $\mathrm{C}$ domain, but the precise mode and architecture of binding in vivo are still unknown. $^{51}$ Our data in vitro implied that the $\beta$-sheet structure might play a crucial role in the IGF-1 ligand/ receptor binding.

\section{Conclusion}

In summary, we reported a cell enhancement strategy with a hybrid $\beta$-sheet peptide hydrogel which combined a biomimetic constituent IGF-1C and a D-form peptide for the first time. This hydrogel favors cell retention, provides a proangiogenic niche for the transplanted cells, and achieves an integrated optimal therapy. This study introduced a novel strategy for integration of self-assembling peptide hydrogel and stem cell therapy, and might shed light into the development of tissue engineering as well as regenerative medicine.

\section{Abbreviations}

AKI, acute kidney injury; ATN, acute tubular necrosis; BLI, bioluminescence imaging; DMEM, Dulbecco Minimum Essential Medium; ECM, extracellular matrix; ESKD, end-stage kidney disease; FBS, fetal bovine serum; Fluc, firefly luciferase; HE, hematoxylin-eosin; hP-MSCs, human placenta-derived mesenchymal stem cells; IGF-1C, $\mathrm{C}$ domain of insulin-like growth factor-1; MSCs, mesenchymal stem cells; NEAA, non-essential amino acid; p/s/ $\mathrm{cm}^{2} / \mathrm{sr}$, photons $/$ second $/ \mathrm{cm}^{2} /$ steradian; PBS, phosphate buffered solution; TEM, transmission electron microscopy; VEGFR2, vascular endothelial growth factor receptor 2 .

\section{Data Sharing Statement}

All data generated and/or analyzed during this study are available from the corresponding author upon reasonable request.

\section{Ethics Approval and Consent to Participate}

The protocols involved animals and the experimental procedures of the present study were approved by the Nankai University Animal Care and Use Committee guidelines that conform to the Guidelines for Animal Care approved by National Institutes of Health (8th Edition, 2011).

\section{Acknowledgment}

The abstract of this paper was presented at the ISN World Congress of Nephrology (WCN) as a poster presentation with interim findings. The poster's abstract was published in 'Poster Abstracts' in Kidney International Reports: https://www.sciencedirect.com/science/article/pii/ S2468024920306021

\section{Author Contributions}

All authors made substantial contributions to conception and design, acquisition of data, or analysis and interpretation of data; took part in drafting the article or revising it critically for important intellectual content; gave final approval of the version to be published; and agree to be accountable for all aspects of the work. 


\section{Funding}

This research was partially supported by National Key R\&D Program of China (2017YFA0103203), Key Project of the National Natural Science Foundation of China (81830060), National Key R\&D Program of China (2018YFA0108803), and Key Projects of Tianjin Science and Technology Support Program (18YFZCSY00010).

\section{Disclosure}

The authors declare that there is no conflict of interest regarding the publication of this paper.

\section{References}

1. He N, Xu Y, Du W, et al. Extracellular matrix can recover the downregulation of adhesion molecules after cell detachment and enhance endothelial cell engraftment. Sci Rep. 2015;5:10902. doi:10.1038/srep10902

2. Prince E, Kumacheva E. Design and applications of man-made biomimetic fibrillar hydrogels. Nat Rev Mater. 2019;4(2):99-115. doi:10.1038/s41578-018-0077-9

3. Tang JD, Mura C, Lampe KJ. Stimuli-responsive, pentapeptide, nanofiber hydrogel for tissue engineering. $J$ Am Chem Soc. 2019;141(12):4886-4899. doi:10.1021/jacs.8b13363

4. Chen J, Zou X. Self-assemble peptide biomaterials and their biomedical applications. Bioactive Materials. 2019;4:120-131. doi:10.1016/j.bioactmat.2019.01.002

5. Niklason LE. Understanding the extracellular matrix to enhance stem cell-based tissue regeneration. Cell Stem Cell. 2018;22(3):302-305. doi:10.1016/j.stem.2018.02.001

6. Koutsopoulos S. Self-assembling peptide nanofiber hydrogels in tissue engineering and regenerative medicine: progress, design guidelines, and applications. J Biomed Mater Res A. 2016;104(4):10021016. doi:10.1002/jbm.a.35638

7. Loo Y, Goktas M, Tekinay AB, Guler MO, Hauser CA, Mitraki A. Selfassembled proteins and peptides as scaffolds for tissue regeneration. $A d v$ Healthc Mater. 2015;4(16):2557-2586. doi:10.1002/adhm.201500402

8. Yokoi H, Kinoshita T, Zhang S. Dynamic reassembly of peptide RADA16 nanofiber scaffold. Proc Natl Acad Sci U S A. 2005;102 (24):8414-8419. doi:10.1073/pnas.0407843102

9. Clarke DE, Pashuck ET, Bertazzo S, Weaver JVM, Stevens MM. Self-healing, self-assembled beta-sheet peptide-poly(gamma-glutamic acid) hybrid hydrogels. J Am Chem Soc. 2017;139(21):72507255. doi:10.1021/jacs.7b00528

10. Elsawy MA, Smith AM, Hodson N, Squires A, Miller AF, Saiani A. Modification of beta-sheet forming peptide hydrophobic face: effect on self-assembly and gelation. Langmuir. 2016;32(19):4917-4923. doi:10.1021/acs.langmuir.5b03841

11. Shang Y, Zhi D, Feng G, et al. Supramolecular nanofibers with superior bioactivity to insulin-like growth factor-I. Nano Lett 2019;19(3):1560-1569. doi:10.1021/acs.nanolett.8b04406

12. Shang Y, Wang Z, Zhang R, et al. A novel thermogel system of selfassembling peptides manipulated by enzymatic dephosphorylation. Chem Commun. 2019;55(35):5123-5126. doi:10.1039/C9CC00401G

13. Jacob RS, Ghosh D, Singh PK, et al. Self healing hydrogels composed of amyloid nano fibrils for cell culture and stem cell differentiation. Biomaterials. 2015;54:97-105. doi:10.1016/j.biomaterials.2015.03.002

14. Ingavle GC, Gehrke SH, Detamore MS. The bioactivity of agarose PEGDA interpenetrating network hydrogels with covalently immobilized RGD peptides and physically entrapped aggrecan. Biomaterials. 2014;35(11):3558-3570. doi:10.1016/j.biomaterials.2014.01.002
15. Leslie-Barbick JE, Saik JE, Gould DJ, Dickinson ME, West JL. The promotion of microvasculature formation in poly(ethylene glycol) diacrylate hydrogels by an immobilized VEGF-mimetic peptide. Biomaterials. 2011;32(25):5782-5789. doi:10.1016/j.biomaterials.2011.04.060

16. Dang LTH, Feric NT, Laschinger C, et al. Inhibition of apoptosis in human induced pluripotent stem cells during expansion in a defined culture using angiopoietin-1 derived peptide QHREDGS. Biomaterials. 2014;35(27):7786-7799. doi:10.1016/j.biomaterials.2014.05.018

17. Feng G, Zhang J, Li Y, et al. IGF-1 C domain-modified hydrogel enhances cell therapy for AKI. J Am Soc Nephrol. 2016;27(8):23572369. doi:10.1681/ASN.2015050578

18. Zhao N, Yue Z, Cui J, et al. IGF-1C domain-modified hydrogel enhances therapeutic potential of mesenchymal stem cells for hindlimb ischemia. Stem Cell Res Ther. 2019;10(1):129. doi:10.1186/ s13287-019-1230-0

19. Bai M, Zhang L, Fu B, et al. IL-17A improves the efficacy of mesenchymal stem cells in ischemic-reperfusion renal injury by increasing Treg percentages by the COX-2/PGE2 pathway. Kidney Int. 2018;93(4):814-825. doi:10.1016/j.kint.2017.08.030

20. Perico L, Morigi M, Rota C, et al. Human mesenchymal stromal cells transplanted into mice stimulate renal tubular cells and enhance mitochondrial function. Nat Commun. 2017;8(1):983. doi:10.1038/ s41467-017-00937-2

21. Pang P, Abbott M, Chang SL, et al. Human vascular progenitor cells derived from renal arteries are endothelial-like and assist in the repair of injured renal capillary networks. Kidney Int. 2017;91(1):129-143. doi:10.1016/j.kint.2016.07.037

22. Little MH, Kairath P. Regenerative medicine in kidney disease. Kidney Int. 2016;90(2):289-299. doi:10.1016/j.kint.2016.03.030

23. Xu L, Li X, Zhang F, Wu L, Dong Z, Zhang D. EGFR drives the progression of AKI to CKD through HIPK2 overexpression. Theranostics. 2019;9(9):2712-2726. doi:10.7150/thno.31424

24. Hao T, Li J, Yao F, et al. Injectable fullerenol/alginate hydrogel for suppression of oxidative stress damage in brown adipose-derived stem cells and cardiac repair. ACS Nano. 2017;7b00221.

25. Choi S-K, Park J-K, Kim J-H, et al. Integrin-binding elastin-like polypeptide as an in situ gelling delivery matrix enhances the therapeutic efficacy of adipose stem cells in healing full-thickness cutaneous wounds. J Controlled Release. 2016;237.

26. Hua Y, Pu G, Ou C, et al. Gd(III)-induced supramolecular hydrogelation with enhanced magnetic resonance performance for enzyme detection. Sci Rep. 2017;7:40172. doi:10.1038/srep40172

27. Luo Z, Wu Q, Yang C, et al. A powerful CD8(+) t-cell stimulating d-tetra-peptide hydrogel as a very promising vaccine adjuvant. $A d v$ Mater. 2017;29(5):1601776. doi:10.1002/adma.201601776

28. Liu J, Liu J, Chu L, et al. Self-assembling peptide of D-amino acids boosts selectivity and antitumor efficacy of 10-hydroxycamptothecin. ACS Appl Mater Interfaces. 2014;6(8):5558-5565. doi:10.1021/ am406007g

29. Luo Z, Yue Y, Zhang Y, et al. Designer D-form self-assembling peptide nanofiber scaffolds for 3-dimensional cell cultures. Biomaterials. 2013;34(21):4902-4913. doi:10.1016/j.biomaterials.2013.03.081

30. Chen S, Zhou A, He B, Zhao W, Chen X, Jiang D. Designer D-form self-assembling peptide scaffolds promote the proliferation and migration of rat bone marrow-derived mesenchymal stem cells. Int $J$ Mol Med. 2017;40(3):679-688. doi:10.3892/ijmm.2017.3056

31. Wang H, Feng Z, Xu B. D-amino acid-containing supramolecular nanofibers for potential cancer therapeutics. Adv Drug Deliv Rev. 2017;110-111:102-111. doi:10.1016/j.addr.2016.04.008

32. He B, Ou Y, Zhou A, et al. Functionalized d-form self-assembling peptide hydrogels for bone regeneration. Drug Des Devel Ther. 2016;10:1379-1388. doi:10.2147/DDDT.S97530

33. Patil SB, Vogtli M, Webb B, et al. Decoupling competing surface binding kinetics and reconfiguration of receptor footprint for ultrasensitive stress assays. Nat Nanotechnol. 2015;10(10):899-907. doi:10.1038/nnano.2015.174 
34. Wang R, Zhang K, Tao H, et al. Molecular imaging of tumor angiogenesis and therapeutic effects with dual bioluminescence. Curr Pharm Biotechnol. 2017;18(5):422-428. doi:10.2174/1389201018666170523 165053

35. Zhou M, Wang L, Su W, et al. Assessment of therapeutic efficacy of liposomal nanoparticles mediated gene delivery by molecular imaging for cancer therapy. J Biomed Nanotechnol. 2012;8(5):742-750. doi:10.1166/jbn.2012.1442

36. Zhang K, Wang C, Wang R, Chen S, Li Z. Dual bioluminescence imaging of tumor progression and angiogenesis. JoVE. 2019; e59763.

37. Yao X, Liu Y, Gao J, et al. Nitric oxide releasing hydrogel enhances the therapeutic efficacy of mesenchymal stem cells for myocardial infarction. Biomaterials. 2015;60:130-140. doi:10.1016/j. biomaterials.2015.04.046

38. Li Z, Hu S, Ghosh Z, Han Z, Wu JC. Functional characterization and expression profiling of human induced pluripotent stem cell- and embryonic stem cell-derived endothelial cells. Stem Cells Dev. 2011;20(10):1701-1710. doi:10.1089/scd.2010.0426

39. Ooi HW, Hafeez S, van Blitterswijk CA, Moroni L, Baker MB. Hydrogels that listen to cells: a review of cell-responsive strategies in biomaterial design for tissue regeneration. Mater Horiz. 2017;4 (6):1020-1040. doi:10.1039/C7MH00373K

40. Zhao Y, Zhu R, Song X, et al. Assembly pathway selection of designer self-assembling peptide and fabrication of hierarchical scaffolds for neural regeneration. ACS Appl Mater Interfaces. 2018;10 (31):26128-26141. doi:10.1021/acsami.8b10764

41. Vardar E, Larsson HM, Engelhardt EM, et al. IGF-1-containing multi-layered collagen-fibrin hybrid scaffolds for bladder tissue engineering. Acta Biomater. 2016;41:75-85. doi:10.1016/j.actbio.2016. 06.010
42. Baserga R. The contradictions of the insulin-like growth factor 1 receptor. Oncogene. 2000;19(49):5574-5581. doi:10.1038/sj. onc. 1203854

43. Zhang K, Zhao X, Chen X, et al. Enhanced therapeutic effects of mesenchymal stem cell-derived exosomes with an injectable hydrogel for hindlimb ischemia treatment. ACS Appl Mater Interfaces. 2018;10(36):30081-30091. doi:10.1021/acsami.8b08449

44. Liu N, Patzak A, Zhang J. CXCR4-overexpressing bone marrowderived mesenchymal stem cells improve repair of acute kidney injury. Am J Physiol Renal Physiol. 2013;305(7):F1064-73. doi:10. 1152/ajprenal.00178.2013

45. Li CD, Zhang WY, Li HL, et al. Mesenchymal stem cells derived from human placenta suppress allogeneic umbilical cord blood lymphocyte proliferation. Cell Res. 2005;15(7):539-547. doi:10.1038/sj. cr.7290323

46. Zhou J, Li J, Du X, Xu B. Supramolecular biofunctional materials. Biomaterials. 2017;129:1-27. doi:10.1016/j.biomaterials.2017.03. 014

47. Luo Z, Zhao X, Zhang S. Structural dynamic of a self-assembling peptide d-EAK16 made of only D-amino acids. PLoS One. 2008;3 (5):e2364. doi:10.1371/journal.pone.0002364

48. Zhao X, Cui K, Li Z. The role of biomaterials in stem cell-based regenerative medicine. Future Med Chem. 2019;11(14):1777-1790. doi:10.4155/fmc-2018-0347

49. Kavran JM, McCabe JM, Byrne PO, et al. How IGF-1 activates its receptor. Elife. 2014;3:e03772. doi:10.7554/eLife.03772

50. Xu Y, Kong GK, Menting JG, et al. How ligand binds to the type 1 insulin-like growth factor receptor. Nat Commun. 2018;9(1):821. doi:10.1038/s41467-018-03219-7
International Journal of Nanomedicine

\section{Publish your work in this journal}

The International Journal of Nanomedicine is an international, peerreviewed journal focusing on the application of nanotechnology in diagnostics, therapeutics, and drug delivery systems throughout the biomedical field. This journal is indexed on PubMed Central, MedLine, CAS, SciSearch ${ }^{\mathbb{}}$, Current Contents ${ }^{\mathbb{R}} /$ Clinical Medicine, $^{-}$

\section{Dovepress}

Journal Citation Reports/Science Edition, EMBase, Scopus and the Elsevier Bibliographic databases. The manuscript management system is completely online and includes a very quick and fair peer-review system, which is all easy to use. Visit http://www.dovepress.com/ testimonials.php to read real quotes from published authors. 